\title{
NONDEGENERATE LINEARIZABLE CENTRES OF COMPLEX PLANAR QUADRATIC AND SYMMETRIC CUBIC SYSTEMS IN $\mathbb{C}^{2}$
}

\author{
C. Christopher and C. Rousseau
}

Abstract

In this paper we consider complex differential systems in the plane, which are linearizable in the neighborhood of a nondegenerate centre. We find necessary and sufficient conditions for linearizability for the class of complex quadratic systems and for the class of complex cubic systems symmetric with respect to a centre.

The sufficiency of these conditions is shown by exhibiting explicitly a linearizing change of coordinates, either of Darboux type or a generalization of it.

\section{Introduction}

This paper originated from the interest of the two authors in isochronous centres ([CD], $[\mathbf{M R T}]$ and $[\mathbf{M M R}])$. Several authors have made a systematic study of the isochronous centres inside certain classes of systems with centre ([CJ], $[\mathbf{L}],[\mathbf{P}],[\mathbf{R T} \mathbf{1}],[\mathbf{D e}],[\mathbf{S a 1}]$ and $[\mathbf{S a 2}])$. The first four papers study isochronous centres inside quadratic systems and cubic systems symmetric with respect to its centre. The work of Devlin $[\mathbf{D e}]$ deals with those centres for which there is an integrating factor $\left(x^{2}+y^{2}\right)^{\alpha}$. The papers by Sabatini give conditions for isochronicity in terms of commuting vector fields. These studies are made possible by the fact that the centre conditions are known in both these cases.

Unfortunately, there are very few "natural" families in which the centre conditions are known, although a number of mechanisms producing strata of centres are well known.

In $[\mathbf{C D}]$ the Kukles family of systems was studied:

$$
\begin{aligned}
& \dot{x}=-y \\
& \dot{y}=x+a_{1} x^{2}+a_{2} x y+a_{3} y^{2}+a_{4} x^{3}+a_{5} x^{2} y+a_{6} x y^{2}+a_{7} y^{3} .
\end{aligned}
$$

2000 Mathematics Subject Classification. 34C, 58F.

This work was supported by NSERC and FCAR. 
An original feature of this work is that, although the centre conditions are not known, it is still possible to find necessary and sufficient conditions for an isochronous centre in this family.

The paper $[\mathbf{C D}]$ brings to light other interesting questions, one of which is the starting point for our investigations here. In looking for the necessary conditions for an isochronous centre in the Kukles system, a new set of conditions was found which cannot be satisfied for real systems in the family. However if we allow the coefficients $a_{i}$ to be complex, then the conditions can be satisfied, and the origin is again isochronous. The notion of isochronicity still makes sense in this context as long as we keep to real time.

In $[\mathbf{C D}]$ it was shown that a singular point is a complex isochronous centre if and only if a whole punctured neighborhood of the point consists entirely of closed trajectories. This interesting result thus gives a geometric characterization of isochronicity. This is completely different from the real case, where any centre can be made isochronous by multiplying by a suitable positive function. In the complex case, multiplying by a non-constant function changes the geometry of the (real time) trajectories.

Simultaneous to these investigations, there has been a growing interest in the complex centres of systems in $\mathbb{C}^{2}$, a centre being a non-degenerate singular point with zero trace and a local analytic first integral. Note that integrable saddles also come within this category. Complex centres in quadratic systems have been studied by several mathematicians: Dulac $[\mathbf{D u}]$, Liu and Li $[\mathbf{L L}]$ and more recently Farell $[\mathbf{F}]$.

Putting together the results of these works it becomes natural to ask what are the complex isochronous quadratic systems. This raises the need of a good definition of a complex isochronous centre. Using a real time for such a system is somewhat artificial, especially since it excludes the natural identification of centres and saddles when working over the complex numbers. Our starting point here, therefore, is to introduce the equivalent notion of linearizable centre. That is, a system whose centre can be reduced to its linear part by an analytic change of coordinates.

In this paper we give necessary and sufficient conditions for linearizability of complex quadratic and symmetric cubic centres. The paper $[\mathbf{M M R}]$ introduces a new method to prove isochronicity via Darboux linearizing change of coordinates. A refinement of this method allows us to give explicit linearizing changes of coordinates for each of these conditions.

One important contribution of these investigations to the study of isochronicity is that there seems to be a far wider range of linearizable 
centres if we allow the coefficients to be complex. This seems to be in contrast to the case of integrability, where the corresponding strata are very similar. However, if we consider linearizable saddles instead, then the complex and real cases are comparable.

A simple reason for this situation is that, for real centres, the separatrices are conjugate and there can be no independence in how a real linearizing transformation can act on either one. In the complex case and in the case of a real saddle this independence is preserved.

It seems, therefore, that a better understanding of isochronicity as a phenomena should be obtained from considering linearizable saddles in more detail.

In all studies of this nature, there is a need to have "visible" phenomena to spur on investigation. In the case of a real isochronous centre, it is easy to see that no finite critical point can lie on the boundary of the period annulus attached to the centre; a result which has been used in several classification problems. If we consider linearizable saddles, then this phenomena only remains valid with complex time, and consequently loses its power. However, from our investigations we conjecture the following obstruction to linearizability, which we hope will provide an impetus to further investigation along these lines: No linearizable saddle can lie on a homoclinic loop or, more generally, on a monodromic graphic.

\section{Generalities}

\subsection{Complex centres and linearizable centres.}

Definition 2.1. (1) A singular point of a complex analytic system

$$
\begin{aligned}
\dot{z} & =P(z, w) \\
\dot{w} & =Q(z, w)
\end{aligned}
$$

in $\mathbb{C}^{2}$ is a centre if the system has an analytic first integral in a neighborhood of the singular point.

(2) A centre is nondegenerate if the system has a non-vanishing 1-jet at the singular point which is a Morse singular point of an analytic first integral.

(3) A nondegenerate centre is linearizable if there exists an analytic change of coordinates in the neighborhood of the singular point, bringing the system to a linear system.

Remark 2.2. A nondegenerate centre necessarily has two opposite eigenvalues. 
From now on, we will always restrict ourselves to nondegenerate centres.

Proposition 2.3. Let us suppose that the system (2.1) has a nondegenerate centre at the origin and is of the form

$$
\begin{aligned}
& \dot{z}=i z+p(z, w)=i z+o\left(|(z, w)|^{2}\right) \\
& \dot{w}=-i w+q(z, w)=-i w+o\left(|(z, w)|^{2}\right) .
\end{aligned}
$$

The following are equivalent:

(1) The origin is linearizable;

(2) There exists a neighborhood of the origin such that every trajectory inside that neighborhood (with time as a real parameter) is periodic (if this is so, then the period is a constant).

(3) The origin is stable, in the sense stable in the future and stable in the past, for real time $t$.

Proof: As pointed by the referee the equivalence of (1) and (3) is a consequence of a theorem of Cartan-CarathThetaodory (1932) (see for instance [M, Theorem 2.1]), stating that a system of the form (2.2) is linearizable if and only if the solution $(z, w)=(0,0)$ is stable. As any trajectory of the linear system is periodic, which implies the same result for the linearizable system, we have that (1) implies (2). The last part follows from (2) implies (3).

Note, that the linearizability condition here is stronger than integrability and is not equivalent to the linearizability of foliations defined by 1-forms. More details on this can be found in [CMR].

\subsection{Darboux linearization and its generalization.}

We start with some definitions.

Definitions 2.4. (1) For equation (2.1) we define the differential operator $D$ acting on analytic functions $F(z, w)$ defined in a neighborhood of $(0,0)$ by

$$
D(F)=\frac{\partial F}{\partial z} P(z, w)+\frac{\partial F}{\partial w} Q(z, w) .
$$

(2) An invariant algebraic curve of the system (2.1) is a curve in $\mathbb{C}^{2}$ given by an equation $F(z, w)=0$, with $F(z, w) \in \mathbb{C}[z, w]$ such that there exists $K(z, w) \in \mathbb{C}_{n-1}[z, w]$ satisfying

$$
D F(z, w)=F(z, w) K(z, w) .
$$


Here $\mathbb{C}_{n-1}[z, w]$ denotes the space of polynomials in $z$ and $w$ of degree $\leq n-1$ and complex coefficients. $F(x, y)$ is also called a Darboux factor.

(3) Any analytic function $F(z, w)$ satisfying (2.4), for some $K(z, w) \in$ $\mathbb{C}_{n-1}[z, w]$, is a generalized Darboux factor. The polynomial $K(F)=$ $K(z, w)$ is called the cofactor of the Darboux factor.

(4) A nonconstant function $F(z, w)$ satisfying $D F(z, w) \equiv 0$ is a first integral.

(5) A Darboux function is a function $Z(z, w)$ of the form

$$
Z=\prod_{j=0}^{k} F_{j}^{\alpha_{j}}, \quad \alpha_{j} \in \mathbb{C},
$$

with $F_{j} \in \mathbb{C}[z, w], j=0, \ldots, k$.

(6) Given a system (2.1) and the differential operator $D$ defined by (2.3), a Darboux function (resp. generalized Darboux function) associated with the system (2.1) is a function $Z$ of the form (2.5), with $F_{j}=0$ invariant algebraic curves (resp. $F_{j}$ Darboux factors), $j=0, \ldots, k$.

(7) A system is (generalized) Darboux integrable if it has a first integral which is a (generalized) Darboux function associated to it.

Many of the strata of polynomial systems with a centre have a first integral which is a Darboux function, or a generalized Darboux function (cf. $[\mathbf{C}],[\mathbf{S 1}],[\mathbf{S 2}])$. In practice, the Darboux factors that we are most interested in arise as limiting cases of Darboux functions, and can be expressed in the form $e^{D / E}$, where $D$ and $E$ are polynomials $[\mathbf{C}]$. Many examples of isochronous centres having Darboux first integrals are also given in [MRT].

The concept of Darboux linearizability is introduced in [MMR], which however is only concerned with real systems. In the real context a linearizing change of coordinates is hence given by a unique function $Z=F(z, \bar{z})$. Here we must adapt the definitions to the fact that we are dealing with systems in $\mathbb{C}^{2}$.

Definition 2.5. The system (2.2) is (generalized) Darboux linearizable if there exists a (generalized) Darboux change of coordinates

$$
(Z, W)=\left(\prod_{j=0}^{k} F_{j}^{\alpha_{j}}, \prod_{j=0}^{\ell} G_{j}^{\beta_{j}}\right),
$$


regular at the origin, i.e. of the form $(Z, W)=(z+o(|(z, w)|), w+$ $o(|(z, w)|))$ linearizing $(2.2)$. Such a function $Z$ is called a (generalized) Darboux linearizing change of coordinates.

Remark 2.6. A (generalized) Darboux linearizable system is (generalized) Darboux integrable with first integral $F(Z, W)=Z W$.

The following theorem characterizing Darboux linearizability is obtained exactly as the corresponding theorem in [MMR]:

Theorem 2.7. $\quad$ (i) The system (2.2) is Darboux linearizable if and only if there exist invariant algebraic curves $F_{0}=0$ and $G_{0}=0$ of the form $F_{0}(z, w)=z+o(|(z, w)|), G_{0}(z, w)=w+o(|(z, w)|)$ and there exist invariant algebraic curves $F_{j}=0, j \in J_{1}, G_{j}=0$, $j \in J_{2}$ where $J_{1}$ and $J_{2}$ are finite subsets of $\mathbb{N}$ (possibly void) such that $F_{j}(0,0) \neq 0, G_{j}(0,0) \neq 0$ and

$$
\begin{aligned}
K_{0}+\sum_{j \in J_{1}} \alpha_{j} K_{j} & =i \\
L_{0}+\sum_{j \in J_{2}} \beta_{j} L_{j} & =-i
\end{aligned}
$$

where $K_{j}$ is the cofactor of $F_{j}, L_{j}$ is the cofactor $G_{j}$ and $\alpha_{j}, \beta_{j} \in$ $\mathbb{C}$. The Darboux linearizing change of coordinates is then given by

$$
(Z, W)=\left(F_{0} \prod_{j \in J_{1}} F_{j}^{\alpha_{j}}, G_{0} \prod_{j \in J_{2}} G_{j}^{\beta_{j}}\right) .
$$

(ii) The system (2.2) is generalized Darboux linearizable if and only if (2.7) is satisfied with the $K_{j}$ and $L_{j}$ cofactors of Darboux factors $F_{j}$ and $G_{j}$. The linearizing change of coordinates is again given by $(2.8)$.

Proof: The proof goes exactly as in [MMR]. It follows from the fact that $(Z, W)=(F(z, w), G(z, w))$ is a linearizing change of coordinates if and only if the functions $F$ and $G$ are Darboux factors satisfying $D F=i$ and $D G=-i$.

It turned out, in several examples of Darboux integrable systems, that we could only find algebraic invariant curves or Darboux factors so that one equation of (2.7) is satisfied. In order to construct the linearizing change of coordinates in that case we prove the following lemma. 
Lemma 2.8. Suppose that the system (2.2) has a first integral $H(z, w)=$ $z w+o\left(|(z, w)|^{2}\right)$, and that there exist (generalized) Darboux factors $F_{j}$ such that the first equation of (2.7) is satisfied. Then a linearizing change of coordinate is given by

$$
(Z, W)=\left(F_{0} \prod_{j \in J_{1}} F_{j}^{\alpha_{j}}, \frac{H(z, w)}{F_{0} \prod_{j \in J_{1}} F_{j}^{\alpha_{j}}}\right) .
$$

Proof: Let us call $F=F_{0} \prod_{j \in J_{1}} F_{j}^{\alpha_{j}}$ and $G=\frac{H(z, w)}{F_{0} \prod_{j \in J_{1}} F_{j}^{\alpha_{j}}}$. Then $H=F G$. Since $H$ is a first integral we have $D H=0$. From $D F=i$ we then deduce that $D G=-i$.

Remark 2.9. A common method to exhibit explicitly a first integral is to use the Darboux method (with invariant algebraic curves or Darboux factors). It may happen that all Darboux factors occuring in the expression of the first integral do not vanish at the origin. In that case the integral obtained $H(z, w)$ does not vanish at the origin. The theorem has to be applied to the first integral $\bar{H}(z, w)=H(z, w)-H(0,0)$. Even if $H$ is a Darboux first integral it may occur that $\bar{H}$, and hence $G$ is not a Darboux function.

\subsection{A class of Darboux linearizable centres.}

Theorem 2.10. The system

$$
\begin{aligned}
\dot{z} & =i z+z^{n}+a w^{n} \\
\dot{w} & =-i w+\frac{1}{n} z^{n-1} w
\end{aligned}
$$

has a linearizable centre at the origin.

Proof: For each system, we have the invariant algebraic curves $F_{1}(z, w)=$ $w=0$ and $F_{2}(z, w)=z-i a w^{n} /(n+1)=0$, with cofactors $-i+$ $z^{n-1} / n$ and $i+z^{n-1}$ respectively. A third invariant curve with cofactor $K_{3}(z, w)=(n-1) z^{n-1}$ is given by $F_{3}(z, w)=1+h\left(z, w^{n}\right)$, where $h(z, W)=\sum_{j=0}^{n-1} a_{j} z^{j} W^{n-1-j}$, with

$$
a_{n-1}=-i, \quad a_{j}=-\frac{i(j+1) a}{n(n-1-j)-j} a_{j+1} \quad j=0, \ldots, n-2 .
$$

The Darboux linearizing change of coordinates is then given by:

$$
(Z, W)=\left(\frac{F_{2}(z, w)}{\left(F_{3}(z, w)\right)^{1 /(n-1)}}, \frac{w}{\left(F_{3}(z, w)\right)^{1 /(n(n-1))}}\right) .
$$




\section{Linearizable complex quadratic systems}

Theorem 3.1. We consider a quadratic system in $\mathbb{C}^{2}$ :

$$
\begin{aligned}
& \dot{z}=i z+c_{20} z^{2}+c_{11} z w+c_{02} w^{2} \\
& \dot{w}=-i w+d_{20} z^{2}+d_{11} z w+d_{02} w^{2} .
\end{aligned}
$$

The system has a linearizable centre if and only if one of the following conditions is satisfied

$$
\begin{array}{rl}
I & c_{11}=d_{20}=d_{11}=0 \\
I I & c_{11}=c_{02}=d_{11}=0 \\
I I I & c_{02}=d_{20}=0, \quad c_{20}-d_{11}=d_{02}-c_{11}=0 \\
I V & 7 c_{11}-6 d_{02}=7 d_{11}-6 c_{20}=7 d_{11}^{2}-12 d_{20} d_{02} \\
& =14 d_{20} c_{02}-3 d_{11} d_{02}=49 d_{11} c_{02}-18 d_{02}^{2}=0 \\
V & 2 c_{20}-5 d_{11}=2 d_{02}-5 c_{11}=15 d_{11}^{2}+4 d_{20} d_{02} \\
& =6 d_{02}^{2}+25 c_{02} d_{11}=10 d_{20} c_{02}-9 d_{11} d_{02}=0 \\
\text { VI } & c_{11}=c_{02}=d_{02}=0 \\
\text { VII } & c_{20}=d_{20}=d_{11}=0 \\
\text { VIII } & c_{11}=d_{20}=d_{02}=c_{20}-2 d_{11}=0 \\
\text { IX } & c_{20}=c_{02}=d_{11}=d_{02}-2 c_{11}=0 .
\end{array}
$$

In all cases one separatrix of the origin is an algebraic curve.

Proof: To prove the necessity of the conditions, we bring the system (3.1) to normal form

$$
\begin{gathered}
\dot{Z}=i Z+\sum_{j \geq 1} c_{j} Z^{j+1} W^{j} \\
\dot{W}=-i W+\sum_{j \geq 1} d_{j} Z^{j} W^{j+1}
\end{gathered}
$$

up to terms of order 7 under a change of coordinates $(z, w)=(Z+$ $o(|Z, W|), W+o(|Z, W|))$. If the system is linearizable then we must have $a_{1}=a_{2}=a_{3}=b_{1}=b_{2}=b_{3}=0$. The computations of $a_{j}$ and $b_{j}$, for $j=1,2,3$ were performed in Maple and Reduce and a factorised Gröbner basis produced. The results were checked carefully between both packages, and yield the conditions I-IX above.

The sufficiency of the conditions is given below: for each case (3.2)(3.10) we give a linearizing change of coordinates. All Darboux factors are noted $F_{i}$ and their respective cofactors $K_{i}$. 
(1) $c_{11}=d_{20}=d_{11}=0$. Let us first suppose that $c_{20}, d_{02} \neq 0$. We then scale $c_{20}=d_{02}=1$. (The case $c_{02}=0$ corresponds to the Loud system $\left(S_{1}\right)$ in the notation of $[\mathbf{M R T}]$.) The system has four invariant lines:

$$
\begin{array}{ll}
F_{1}(z, w)=w & K_{1}(z, w)=-i+w \\
F_{2}(z, w)=1+i w & K_{2}(z, w)=w \\
F_{3,4}=1-i z+B_{3,4} w & K_{3,4}(z, w)=z-i B_{3,4} w
\end{array}
$$

where $B_{3,4}$ are the roots of $B^{2}-i B-c_{02}=0$. The first integral is given by

$$
H(z, w)=\frac{1-i z+B_{3} w}{1-i z+B_{4} w}(1+i w)^{i\left(B_{3}-B_{4}\right)} .
$$

Choosing $\bar{H}(z, w)=i \frac{1-H(z, w)}{B_{3}-B_{4}}=z w+o\left(|z, w|^{2}\right)$, the linearizing change of coordinates is given by

$$
(Z, W)=\left(\frac{\bar{H}(z, w)(1+i w)}{w}, \frac{w}{1+i w}\right)
$$

We now consider the case $d_{02}=0$ and $c_{20}=1$ (after scaling). Darboux factors and cofactors are given by:

$$
\begin{array}{ll}
F_{1}(z, w)=w & K_{1}(z, w)=-i \\
F_{2,3}(z, w)=1-i z \pm w & K_{2,3}(z, w)=z \mp i w \\
F_{4}(z, w)=e^{w} & K_{4}(z, w)=-i w .
\end{array}
$$

This yields a first integral

$$
H(z, w)=e^{-2 w} \frac{1-i z+w}{1-i z-w} .
$$

We let $\bar{H}(z, w)=\frac{1}{2 i}(H(z, w)-1)=z w+o\left(|z, w|^{2}\right)$ yielding the linearizing change of coordinates

$$
(Z, W)=\left(\frac{\bar{H}(z, w)}{w}, w\right) .
$$

The case $c_{20}=d_{02}=0$ is contained in (7) below.

(2) $\quad c_{11}=c_{02}=d_{11}=0$ is dual of (1) under $(z, w, t) \mapsto(w, z,-t)$.

(3) $\quad c_{02}=d_{20}=0, \quad c_{20}-d_{11}=d_{02}-c_{11}=0$. In the case $c_{20} d_{02} \neq 0$ we can scale $c_{20}=d_{02}=1$, yielding the Loud system $\left(S_{2}\right)$ in the notation 
of $[$ MRT] . This system has the three invariant lines:

$$
\begin{array}{ll}
F_{1}(z, w)=z & K_{1}(z, w)=i+z+w \\
F_{2}(z, w)=w & K_{2}(z, w)=-i+z+w \\
F_{3}=1-i z+i w & K_{3}(z, w)=z+w,
\end{array}
$$

yielding the linearizing change of coordinates

$$
(Z, W)=\left(\frac{z}{1-i z+i w}, \frac{w}{1-i z+i w}\right) .
$$

In the nonlinear case with $c_{20}=0$ or $d_{02}=0$ we are in case (7) or (6) below.

(4) $7 c_{11}-6 d_{02}=7 d_{11}-6 c_{20}=7 d_{11}^{2}-12 d_{20} d_{02}=14 d_{20} c_{02}-3 d_{11} d_{02}=$ $49 d_{11} c_{02}-18 d_{02}^{2}=0$. Either $c_{20}$ and $d_{02}$ vanish simultaneously, in which case we have a subcase of (6) or (7), or they are both nonzero, yielding the Loud system $\left(S_{4}\right)$ (in the notation of [MRT]), which can be scaled to

$$
\begin{aligned}
\dot{z} & =i z+7 z^{2}+6 z w+3 w^{2} \\
\dot{w} & =-i w+3 z^{2}+6 z w+7 w^{2} .
\end{aligned}
$$

This system has an invariant line and three invariant conics:

$$
\begin{aligned}
F_{1}(z, w) & =1-4 i(z-w) & & K_{1}(z, w)=4(z+w) \\
F_{2}(z, w) & =1-16 i(z-w) & & K_{2}(z, w)=16(z+w) \\
& -96\left(z^{2}+w^{2}\right)-64 z w & & \\
& F_{3}(z, w)=z-i(z-w)^{2} & & K_{3}(z, w)=i+8(z+w) \\
F_{4}(z, w) & =w+i(z-w)^{2} & & K_{4}(z, w)=-i+8(z+w),
\end{aligned}
$$

yielding the linearizing change of coordinates

$$
(Z, W)=\left(\frac{z-i(z-w)^{2}}{(1-4 i(z-w))^{2}}, \frac{w+i(z-w)^{2}}{(1-4 i(z-w))^{2}}\right) .
$$

(5) $\quad 2 c_{20}-5 d_{11}=2 d_{02}-5 c_{11}=15 d_{11}^{2}+4 d_{20} d_{02}=6 d_{02}^{2}+25 c_{02} d_{11}=$ $10 d_{20} c_{02}-9 d_{11} d_{02}=0$. Here again $c_{20}$ and $d_{02}$ vanish simultaneously, a case treated in (6) or (7). The nonvanishing case corresponds to the Loud system $\left(S_{3}\right)$ (in the notation of $[\mathbf{M R T}]$ ) and can be scaled to

$$
\begin{aligned}
\dot{z} & =i z+5 z^{2}+2 z w-3 w^{2} \\
\dot{w} & =-i w-3 z^{2}+2 z w+5 w^{2} .
\end{aligned}
$$


This system has an invariant line and two invariant conics:

$$
\begin{array}{ll}
F_{1}(z, w)=1-8 i(z-w) & K_{1}(z, w)=8(z+w) \\
F_{2}(z, w)=z+i(z+w)^{2} & K_{2}(z, w)=i+4(z+w) \\
F_{3}(z, w)=w-i(z+w)^{2} & K_{3}(z, w)=-i+4(z+w) .
\end{array}
$$

The linearizing change of coordinates is given by

$$
(Z, W)=\left(\frac{z+i(z+w)^{2}}{\sqrt{1-8 i(z-w)}}, \frac{w-i(z+w)^{2}}{\sqrt{1-8 i(z-w)}}\right) .
$$

(6) $\quad c_{11}=c_{02}=d_{02}=0$. When $c_{20} \neq 0$ we scale $c_{20}=1$. Scaling on $w$ allows to choose $d_{20}=0,1$. In the case $d_{20}=0$ the system is linearized via

$$
(Z, W)=\left(\frac{z}{1-i z}, \frac{w}{(1-i z)^{d_{11}}}\right) .
$$

If $d_{20}=1$, the system has two invariant lines and an invariant conic:

$$
\begin{array}{rlrl}
F_{1}(z, w) & =z & & K_{1}(z, w)=i+z \\
F_{2}(z, w) & =1-i z & & K_{2}(z, w)=z \\
F_{3}(z, w) & =-2+2 i\left(1+d_{11}\right) z & & K_{3}(z, w)=\left(1+d_{11}\right) z \\
& +\left(d_{11}+d_{11}^{2}\right) z^{2} & & \\
& +d_{11}\left(d_{11}^{2}-1\right) z w &
\end{array}
$$

yielding first integrals

$$
H(z, w)=F_{3}(z, w)(1-i z)^{-\left(1+d_{11}\right)}
$$

and $\bar{H}(z, w)=\frac{1}{d_{11}\left(d_{11}^{2}-1\right)}(H(z, w)-H(0,0))=z(w+o(|(z, w)|))$. The linearizing change of coordinates is given by

$$
(Z, W)=\left(\frac{z}{1-i z}, \frac{\bar{H}(z, w)(1-i z)}{z}\right) .
$$

We next consider the case $c_{20}=0$. If $d_{11} \neq 0$ we scale $d_{11}=1$. If $d_{20}=0$ we have two invariant lines $z=0, w=0$ and the Darboux factor $e^{z}$, yielding the linearizing change of coordinates

$$
(Z, W)=\left(z, w e^{i z}\right) .
$$


In the case $c_{20}=0 d_{11}=1$ and $d_{20} \neq 0$ the system has Darboux factors

$$
\begin{array}{ll}
F_{1}(z, w)=z & K_{1}(z, w)=i \\
F_{2}(z, w)=1-i z-\frac{1}{2} z^{2}-\frac{1}{2 d_{20}} z w & K_{2}(z, w)=z \\
{[4 p t] F_{3}(z, w)=e^{z}} & K_{3}(z, w)=i z .
\end{array}
$$

This yields the first integral

$$
H(z, w)=e^{i z}\left(1-i z-\frac{1}{2} z^{2}-\frac{1}{2 d_{20}} z w\right)
$$

We consider the first integral $\bar{H}(z, w)=-2 d_{20}(H(z, w)-1)=w z+$ $o\left(|z, w|^{2}\right)$, yielding the linearizing change of coordinates

$$
(Z, W)=\left(z, \frac{\bar{H}(z, w)}{z}\right)
$$

If $c_{20}=d_{11}=0$ we have a Hamiltonian system linearized via

$$
(Z, W)=\left(z, w+\frac{i}{3} z^{2}\right)
$$

(7) $\quad c_{20}=d_{20}=d_{11}=0$. This case is the dual of (6).

(8) and (9) are special cases of Theorem 2.10.

\section{Linearizable complex cubic symmetric systems}

Theorem 4.1. We consider a complex cubic symmetric system in $\mathbb{C}^{2}$

$$
\begin{aligned}
& \dot{z}=i z+c_{30} z^{3}+c_{21} z^{2} w+c_{12} z w^{2}+c_{03} w^{3} \\
& \dot{w}=-i w+d_{30} z^{3}+d_{21} z^{2} w+d_{12} z w^{2}+d_{03} w^{3} .
\end{aligned}
$$

The system has a linearizable centre if and only if

$$
c_{21}=d_{12}=0
$$

and one of the following conditions is satisfied 


$$
\begin{array}{rl}
I & c_{30}=d_{30}=d_{21}=0 \\
I I & c_{12}=c_{03}=d_{03}=0 \\
I I I & c_{30}-d_{21}=c_{12}-d_{03}=d_{30}=c_{03}=0 \\
I V & c_{12}=c_{03}=d_{30}=d_{21}=0 \\
V & 3 c_{30}-7 d_{21}=3 d_{03}-7 c_{12}=48 c_{30}^{3}+343 c_{12} d_{30}^{2} \\
& =16 c_{30} c_{12}-21 d_{30} c_{03}=49 c_{12}^{2} d_{30}+9 c_{30}^{2} c_{03} \\
& =27 c_{30} c_{03}^{2}+112 c_{12}^{3}=0 \\
V I & d_{30}=d_{03}=c_{12}=c_{30}-3 d_{21}=0 \\
\text { VII } & c_{30}=c_{03}=d_{21}=d_{03}-3 c_{12}=0 .
\end{array}
$$

In all cases one separatrix of the origin is an algebraic curve.

Proof: The necessity of the conditions is obtained by setting to zero the coefficients of the normal form (3.11) up to degree 7 and the sufficiency by providing the linearizing change of coordinates. As there are no new arguments we limit ourselves to the generic cases. The limiting cases can be done as in Section 3 (see also $[\mathbf{C}]$ ).

(1) $\quad c_{30}=d_{30}=d_{21}=0$. If $d_{03} \neq 0$ we can scale $d_{03}=i$. The system has the three invariant lines $F_{1}(z, w)=w=0$ and has $F_{2,3}(z, w)=1 \pm$ $w=0$ with respective cofactors $K_{1}(z, w)=-i\left(1-w^{2}\right)$ and $K_{2,3}(z, w)=$ $i w(w \mp 1)$.

If $c_{03} \neq 0$ we can scale $c_{03}=1$. We then have for $c_{12} \neq \pm i$ an invariant conic $F_{4}(z, w)=1+\frac{i}{2}\left(1+c_{12}^{2}\right) z w+\frac{1}{2}\left(-1+i c_{12}\right) w^{2}$ with cofactor $K_{4}(z, w)=\left(i+c_{12}\right) w^{2}$. This yields a Darboux first integral

$$
H(z, w)=\frac{\left(1-w^{2}\right)^{1-i c_{12}}}{F_{4}^{2}} .
$$

A linearizing change of coordinates is given by

$$
(Z, W)=\left(\frac{i(H(z, w)-1)\left(1-w^{2}\right)^{1 / 2}}{\left(1+c_{12}^{2}\right) w}, \frac{w}{\left(1-w^{2}\right)^{1 / 2}}\right) .
$$

(2) $\quad c_{12}=c_{03}=d_{03}=0$ is the dual of (1).

(3) $\quad c_{30}-d_{21}=c_{12}-d_{03}=d_{30}=c_{03}=0$. When $c_{30} d_{03} \neq 0$ we recover the case $\left(S_{2}^{*}\right)$ of $[\mathbf{M R T}]$. We can scale $c_{30}=d_{03}=i$, i.e. we have four invariant lines given by $1+i r z+s w=0$ with cofactors of the 
form $-(r z+s w)+i\left(w^{2}+z^{2}\right)$ and $r, s= \pm 1$. This yields a linearizing change of coordinates

$$
(Z, W)=\left(\frac{z}{\sqrt{\left.1-(i z+w)^{2}\right)}}, \frac{w}{\sqrt{\left.1-(i z+w)^{2}\right)}}\right) .
$$

If either $c_{30}=0$ or $d_{03}=0$, we are in case (1) or (2).

(4) $\quad c_{12}=c_{03}=d_{30}=d_{21}=0$. If $c_{30} d_{03} \neq 0$ we recover the case $\left(S_{1}^{*}\right)$ of [MRT]. We can scale $c_{30}=-i$ and $d_{03}=i$. This yields the linearizing change of coordinates

$$
(Z, W)=\left(\frac{z}{\sqrt{1-z^{2}}}, \frac{w}{\sqrt{1-w^{2}}}\right) .
$$

If $c_{30}=0$ or $d_{03}=0$ then we are in case (1) or (2).

(5) $\quad 3 c_{30}-7 d_{21}=3 d_{03}-7 c_{12}=48 c_{30}^{3}+343 c_{12} d_{30}^{2}=16 c_{30} c_{12}-$ $21 d_{30} c_{03}=49 c_{12}^{2} d_{30}+9 c_{30}^{2} c_{03}=27 c_{30} c_{03}^{2}+112 c_{12}^{3}=0$. When $c_{30} d_{03} \neq$ 0 we recover the two cases $\left(S_{3}^{*}\right)$ and $\left(\tilde{S}_{3}^{*}\right)$ of $[$ MRT]. We can scale $c_{30}=7 i=-d_{03}$. This yields $c_{03}=-d_{30}$ and $d_{30}^{2}=-16$. The two cases $d_{30}= \pm 4 i$ are equivalent under $(z, w) \mapsto(z,-w)$. Hence we limit ourselves to the case $d_{30}=4 i$. This system has the first integral

$$
H=\frac{\left(z+(z-w)^{3}\right)\left(w-(z-w)^{3}\right)}{1+9\left(z^{2}+z w+w^{2}\right)},
$$

where the cofactors of the three brackets are $i+9 i\left(z^{2}-w^{2}\right),-i+9 i\left(z^{2}-\right.$ $\left.w^{2}\right)$ and $18 i\left(z^{2}-w^{2}\right)$. From this we obtain the linearising change of coordinates

$$
\begin{aligned}
Z & =\frac{z+(z-w)^{3}}{\sqrt{1+9\left(z^{2}+z w+w^{2}\right)}} \\
W & =\frac{w-(z-w)^{3}}{\sqrt{1+9\left(z^{2}+z w+w^{2}\right)}} .
\end{aligned}
$$

If either $c_{30}=0$ or $d_{03}=0$ we are in case (1) or (2).

(6) $\quad d_{30}=d_{03}=c_{12}=c_{30}-3 d_{21}=0$. If $c_{30}=0$ then we are in case (1), otherwise we scale to make $c_{30}=1$. This is a special case of Theorem 2.10

(7) $\quad c_{30}=c_{03}=d_{21}=d_{03}-3 c_{12}=0$ is the dual of (6). 


\section{Real linearizable saddles}

We now study real quadratic and symmetric cubic systems with a linearizable saddle at the origin. The idea is simply to change coordinates $(z, w, t) \mapsto(i x, i y,-i t)$ in Theorems 3.1 and 4.1. The conditions for integrability given in those theorems thus remain unchanged. This is in marked contrast to the real centre case, where the change of coordinates $(z, w, t) \mapsto(x+i y, x-i y, t)$ forces the conditions to have complex conjugate coefficients and in this way reduces the number of branches that can be realized in $\mathbb{R}^{2}$.

The above observation seems to indicate that the linearizable saddles in some sense give a better indication of what happens in questions of linearizability than is the case for centres. However, the disadvantage is that we lose the visible aspects of the isochronicity, since the system is isochronous with respect to pure imaginary time. Even the concept of an integrable saddle which corresponds to a centre is less well known, and not geometrically intuitive.

All isochronous centres of real quadratic and symmetric cubic systems lie in the stratum of reversible systems. We address the same question for linearizable saddle points to see in which strata of integrable saddles they lie in. Moreover we give their phase portraits to see if there are any general conclusions we can draw on their topology.

It was remarked by Teixeira and Yang $[\mathbf{T Y}]$ that analytic time-reversibility and integrability were equivalent for real centres. We adapt this criterion for our case. The converse part requires more work since we cannot simply assert that time-reversibility implies the existence of an analytic first integral.

Theorem 5.1. A non-degenerate saddle of a real or complex analytic system is integrable if and only if there is a local analytic transformation $T$ of the system, with $T^{2}=\mathrm{id}$ whose effect is the same as the time reversal $t \mapsto-t$.

Proof: Suppose the system is integrable. Then there is an analytic change of coordinates which brings the system to the form

$$
\dot{x}=x\left(\lambda+\sum a_{i}(x y)^{i}\right), \quad \dot{y}=-y\left(\lambda+\sum a_{i}(x y)^{i}\right),
$$

(see for example $[\mathbf{S M}]$ ). It is easy to see that the transformation $(x, y) \mapsto$ $(y, x)$ reverses the system. 
Conversely suppose that the saddle point of the system is not integrable. Modulo an analytic change of coordinates and division by a locally non-vanishing function we can bring the system to a normal form

$$
\dot{x}=x, \quad \dot{y}=-y+A x^{k} y^{k+1}+o\left(|(x, y)|^{2 k+1}\right)
$$

with $A \neq 0$. We will see that this is an obstruction to find an involution. Indeed it can be argued that such an involution $T$ has a linear part of the form $T_{1}(x, y)=(b y, x / b)$, with $b \neq 0$. We look for the involution as a power series $T(x, y)=(X, Y)=\left(b y+\sum_{r=2}^{\infty} h_{r}(x, y)\right.$, $\left.x / b+\sum_{r=2}^{\infty} k_{r}(x, y)\right)$, where $h_{r}(x, y)$ and $k_{r}(x, y)$ are homogeneous polynomials in $x$ and $y$ of degree $r$. From the hypothesis we must have the relation $\dot{X}=-X$. However the term in $x^{k} y^{k+1}$ of degre $2 k+1$ in the expression $\dot{X}+X$ is always of the form $b A \neq 0$, which contradicts the existence of such a $T$.

Remarks 5.2. (1) In fact the hypothesis of the theorem can be relaxed. For example, by considering the order $(2 k+1)$-terms of equation $\dot{Y}$ as well as $\dot{X}$ we can assume only that the linear parts of $T$ (in the expansion about the critical point) are involutive and that the transformed system is equal to the original system multiplied by some negative function. Details are left to the reader.

(2) It is also possible to prove the converse part of the theorem directly, without using normal forms. We sketch the idea below.

If $T:(x, y) \mapsto\left(T_{1}(x, y), T_{2}(x, y)\right)$, then we take new variables $\phi(x, y)=(X, Y)=\left(T_{1}(x, y)-x, T_{1}(x, y)+x\right)$. We want to show that in the coordinates $(X, Y)$ the involution becomes $S(X, Y)=$ $(-X, Y)$, with $S=\phi \circ T \circ \phi^{-1}$. If we call $R(X, Y)=(-X, Y)$ the symmetry with respect to the $Y$-axis, this amounts to showing that $R \circ \phi \circ T(x, y)=\phi(x, y)$, which is a straightforword consequence of the fact that $T$ is an involution.

We thus obtain a new system with a corresponding reversing transformation $S$, and so the system must be of the form

$$
\dot{X}=-Y-P\left(X^{2}, Y\right), \quad \dot{Y}=-X+X Q\left(X^{2}, Y\right) .
$$

Now such a system arises from the system

$$
\dot{W}=-2 Y-2 P(W, Y), \quad \dot{Y}=-1+Q(W, Y),
$$

via the transformation $W=X^{2}$. Since this later system is nonsingular at the origin, it has a first integral $H(W, Y)=W-$ $Y^{2}+o(W)+o\left(Y^{2}\right)$, which can be pulled back to a first integral $K(X, Y)=X^{2}-Y^{2}+o\left(|(X, Y)|^{2}\right)$ of the original system. 
Theorem 5.3. We consider a quadratic system in $\mathbb{R}^{2}$ with a saddle point at the origin with opposite eigenvalues

$$
\begin{aligned}
& \dot{x}=x+c_{20} x^{2}+c_{11} x y+c_{02} y^{2} \\
& \dot{y}=-y+d_{20} x^{2}+d_{11} x y+d_{02} y^{2} .
\end{aligned}
$$

The system is linearizable at the origin if and only if one of the conditions (3.2)-(3.10) is satisfied. The phase portraits are given in Figures $1-6$.

Proof: The system (5.5) is obtained from (3.1) by means of the transformation $(x, y, T)=(-i z,-i w, i t)$.

We have a real system whenever the $c_{j k}$ are real. As the conditions are invariant under $(x, y) \mapsto(a x, b y)$, the families I, II, VI-IX can be reduced to one-dimensional families, while the cases III-V can be reduced to 0-dimensional families. However for practical reasons it is simpler for cases I, II, VI and VII to draw the bifurcation diagram on one fourth of a 2-sphere.

For case I we suppose $c_{20}^{2}+c_{02}^{2}+d_{02}^{2}=1$ and $c_{20}, d_{02} \geq 0$. The bifurcation diagram appears in Figure 1. The case II can be deduced easily from it.

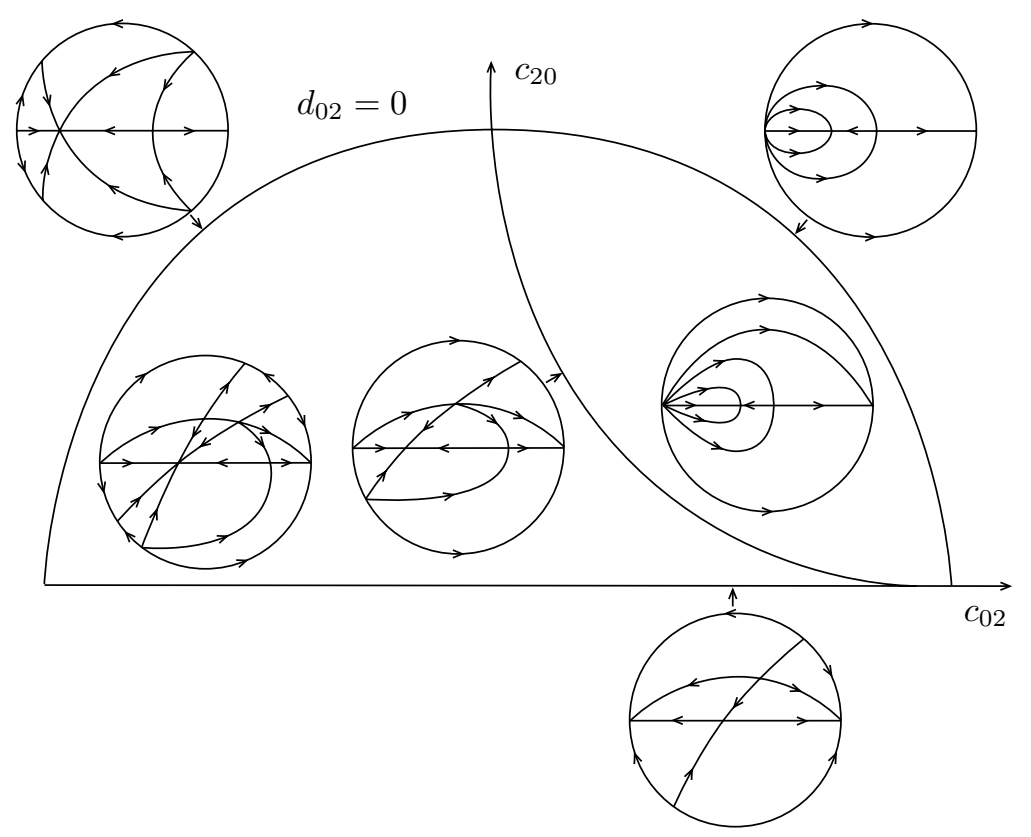

FIGURE 1 
The phase portraits of case III, IV, V appear in Figures 2, 3, and 4 respectively.

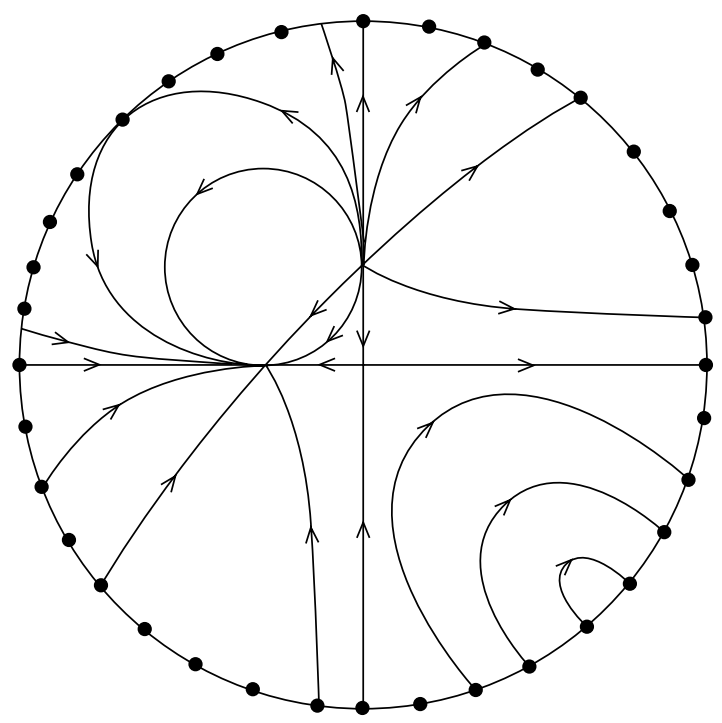

FiguRe 2

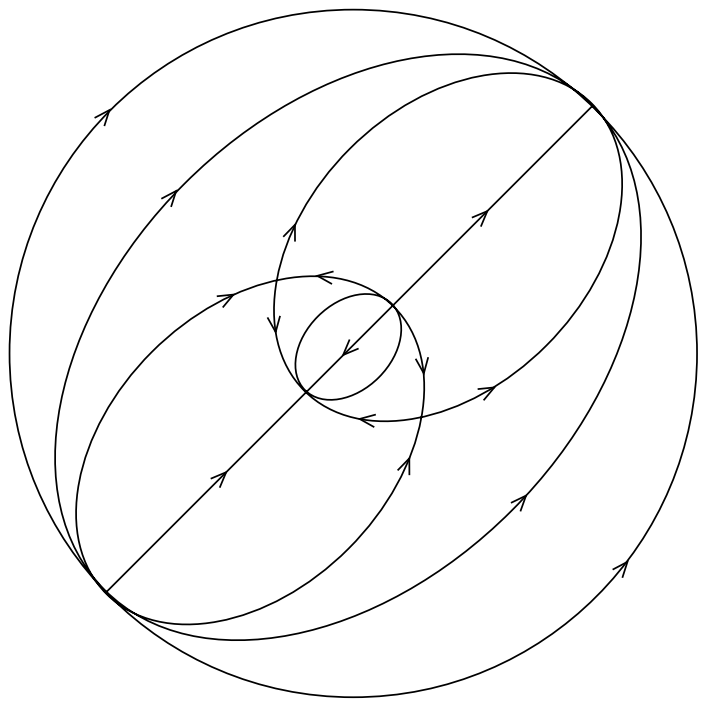

Figure 3 


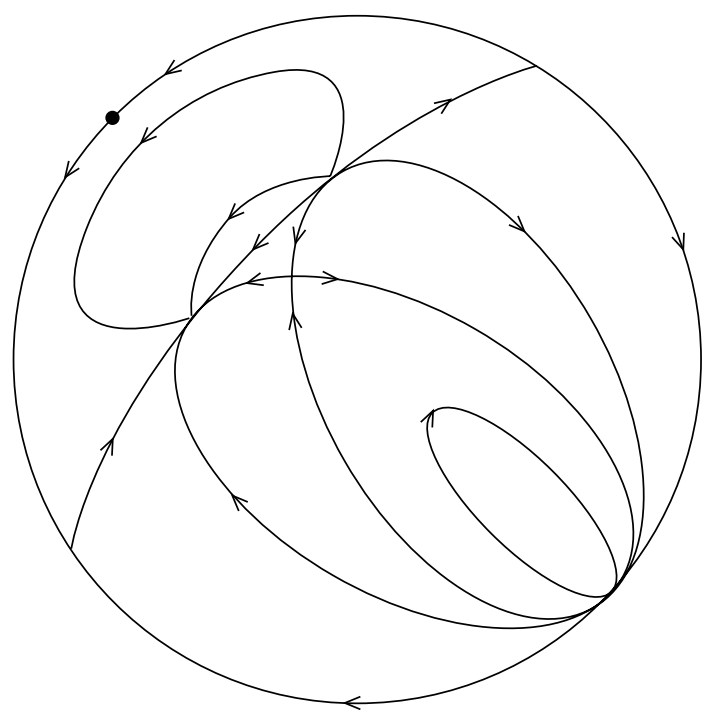

Figure 4

For case VI we suppose $c_{20}^{2}+d_{20}^{2}+d_{11}^{2}=1, c_{20}, d_{20} \geq 0$. The bifurcation diagram appears on Figure 5. Case VII can be easily deduced from it.

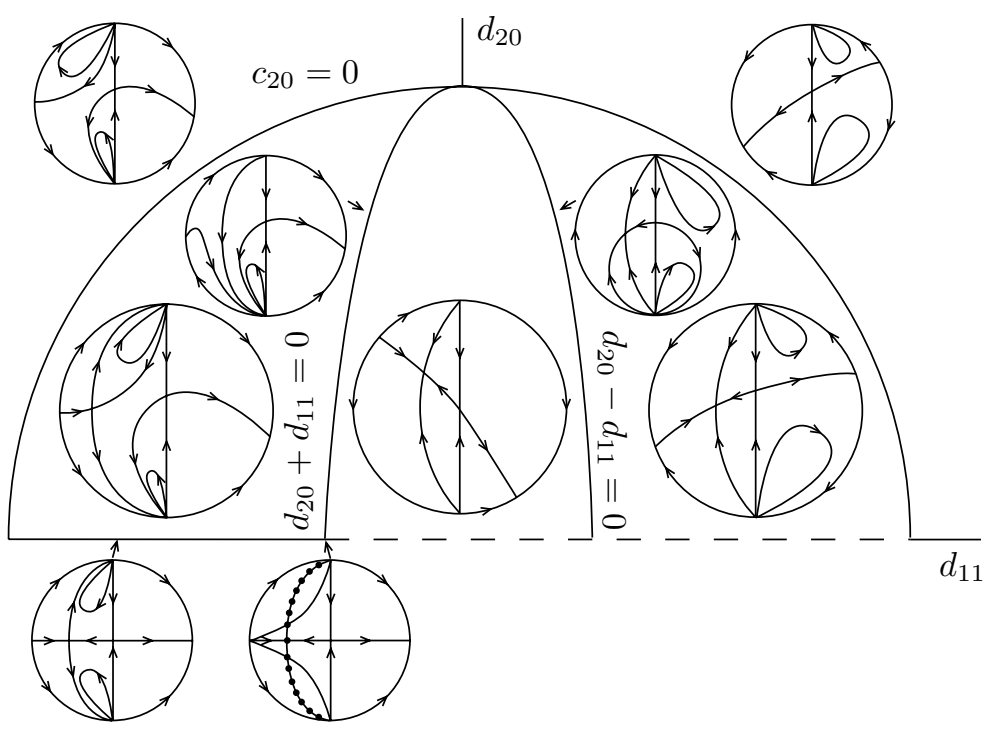

Figure 5 
For case VIII we can scale $d_{11}^{2}+c_{02}^{2}=1, d_{11} \geq 0$. The bifurcation diagram appears in Figure 6. Case IX follows from it.

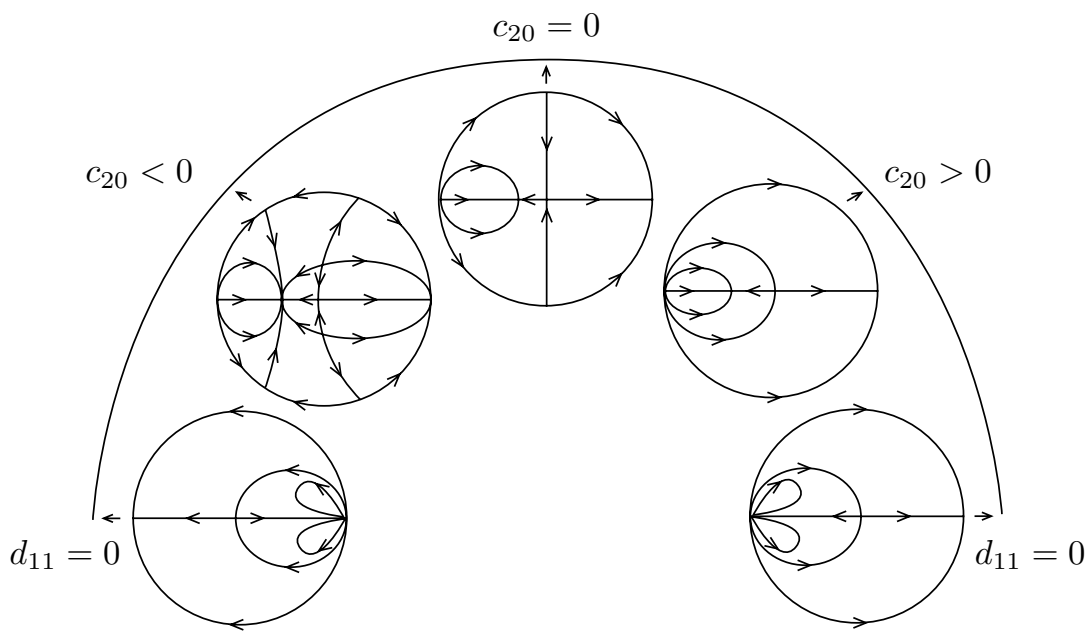

Figure 6

A natural question for us was to see where these linearizable saddles lie inside the strata of integrable saddles. The integrability conditions for a quadratic system with a saddle at the origin first appear in the work of Dulac $[\mathbf{D u}]$ in a case by case procedure. They have been simplified in $[\mathbf{L L}]$ and then further more in $[\mathbf{Z}]$. The phase portraits of integrable saddle points of quadratic systems are systematically studied in [DGS].

Theorem 5.4 ([LL] and $[\mathbf{Z}])$. The strata of integrable saddles of (5.5) are given by

$$
\begin{aligned}
& \text { (A) } c_{11}=d_{11}=0, \\
& \text { (B) } d_{11}+2 c_{20}=c_{11}+2 d_{02}=0, \\
& \text { (C) } c_{20} c_{11}-d_{11} d_{02}=c_{11}^{3} d_{20}-d_{11}^{3} c_{02}=0, \\
& \text { (D) } 2 c_{11}-d_{02}=c_{20}-2 d_{11}=c_{02} d_{20}-d_{11} c_{11}=0 .
\end{aligned}
$$

The stratum $(A)$ consists of systems generically having three invariant lines, allowing a Darboux first integral (called Lotka-Volterra in $[\mathbf{Z}]$ and harmonic in $[\mathbf{L L}])$. 
The stratum $(B)$ consists of Hamiltonian systems.

The stratum $(C)$ consists of systems generically having an invariant line and an invariant conic allowing a Darboux first integral. It is called "symmetric" in $[\mathbf{L L}]$ (for reasons of symmetry in the calculations of Lyapunov quantities) and reversible in $[\mathbf{Z}]$.

The stratum $(D)$ consists of systems having a Malkin first integral generically constructed from an invariant conic and an invariant cubic.

Remark 5.5. When $c_{11} d_{11} \neq 0$ it is not possible to find a symmetry axis in the usual sense and we must introduce a generalization of reversibility, in the same way as exponential factors appear as the limits of Darboux integrals.

The effect of approaching these limiting cases within the stratum $(C)$ is that the eigenvectors of the reversing transformation coalesce and the transformation becomes singular. Thus, in order to resolve this difficulty, we are led to consider the system as a blow-up of a simpler system.

For example the substratum $\left(C_{1}\right)$ of $(C)$ :

$$
\begin{aligned}
& \dot{x}=x+c_{20} x^{2} \\
& \dot{y}=-y+d_{20} x^{2}+d_{11} x y,
\end{aligned}
$$

is a particular case of the following theorem which has been obtained by an algebraic method in $[\mathbf{L L}]$ (symmetries in the calculations of Lyapunov constants).

Theorem 5.6. The following class of integrable saddles lies in the closure of the strata of reversible systems:

$$
\begin{aligned}
& \dot{x}=x+P(x, y)=x(1+p(x, y)) \\
& \dot{y}=-y+Q(x, y),
\end{aligned}
$$

where we have either

$$
P(x, y)=\sum_{\substack{i>j \\ i+j \geq 2}} c_{i j} x^{i} y^{j}, \quad Q(x, y)=\sum_{\substack{i>j-2 \\ i+j \geq 2}} d_{i j} x^{i} y^{j}
$$

with $c_{r+1, r}+d_{r, r+1}=0$, or the conjugate system under $(x, y, t) \mapsto$ $(y, x,-t)$. 
Proof: Taking $X=x$ and $Y=x y$, we obtain the system

$$
\begin{aligned}
& \dot{X}=X\left(1+p\left(X, \frac{Y}{X}\right)\right) \\
& \dot{Y}=Y p\left(X, \frac{Y}{X}\right)+X Q\left(X, \frac{Y}{X}\right) .
\end{aligned}
$$

The conditions imply that a common factor of $X$ can be removed to leave an analytic system with a non critical point at the origin. It therefore has a local first integral $\Phi(X, Y)=Y+o(|(X, Y)|)$ which can be pulled back to a local first integral of the original system, $\Phi(x, x y)=x y+$ $o\left(|(x, y)|^{2}\right)$.

Remarks 5.7. (1) In the corresponding case for real centres, such systems do not arise except in the trivial case

$$
\begin{aligned}
& \dot{x}=-y\left(1+\sum \alpha_{i}\left(x^{2}+y^{2}\right)^{i}\right) \\
& \dot{y}=x\left(1+\sum \alpha_{i}\left(x^{2}+y^{2}\right)^{i}\right) .
\end{aligned}
$$

(2) It would be interesting to see if the corresponding notion of a limit of rational reversible systems would also give some new integrability conditions.

Proposition 5.8. The linearizable saddles described in Theorem 5.3 lie in the stratum $(A)$ for the cases $I-I I$, the stratum $(C)$ for the cases III-VII, and the stratum (D) for the cases VIII-IX. In the latter case the invariant cubic is reducible, yielding a line through the origin and a conic.

Theorem 5.9. We consider a cubic system in $\mathbb{R}^{2}$ symmetric with respect to a saddle point at the origin with opposite eigenvalues

$$
\begin{aligned}
& \dot{x}=x+c_{30} x^{3}+c_{21} x^{2} y+c_{12} x y^{2}+c_{03} y^{3} \\
& \dot{y}=-y+d_{30} x^{3}+d_{21} x^{2} y+d_{12} x y^{2}+d_{03} y^{3} .
\end{aligned}
$$

The system is linearizable at the origin if and only if (4.2) and one of the conditions (4.3)-(4.9) is satisfied. The phase portraits appear in Figures 7-12 (only the generic cases).

Proof: For case I we suppose $c_{12}^{2}+c_{03}^{2}+d_{03}^{2}=1, c_{03} \geq 0$, which yields a half 2 -sphere. The bifurcation diagram appears in Figure 7. (Case II is dual.) 


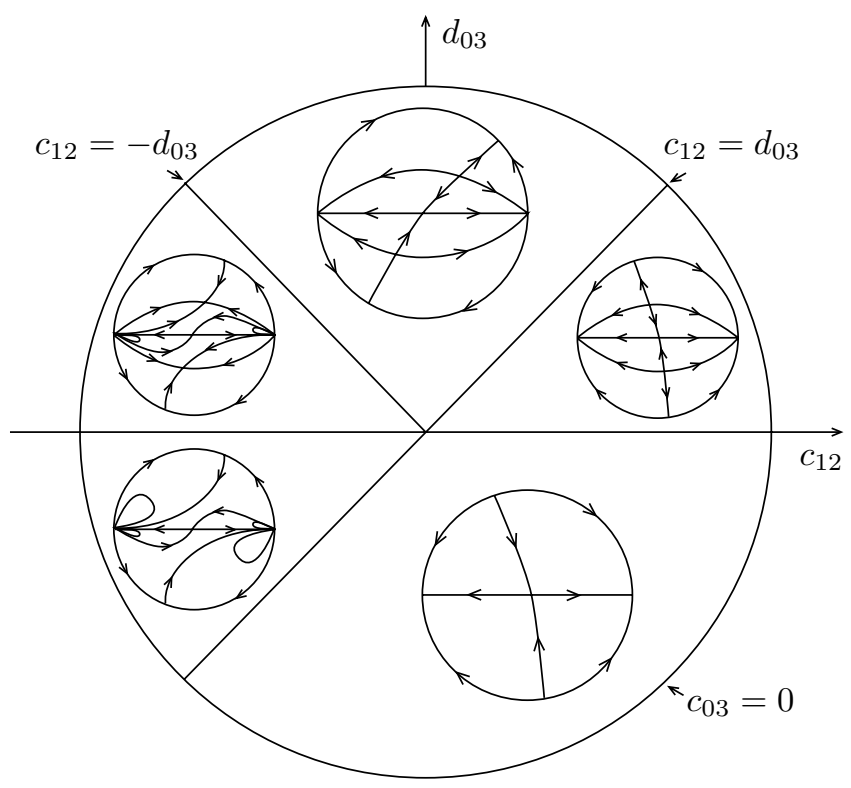

FiguRe 7

For case III we suppose $c_{30}^{2}+c_{12}^{2}=1$. The bifurcation diagram appears in Figure 8.

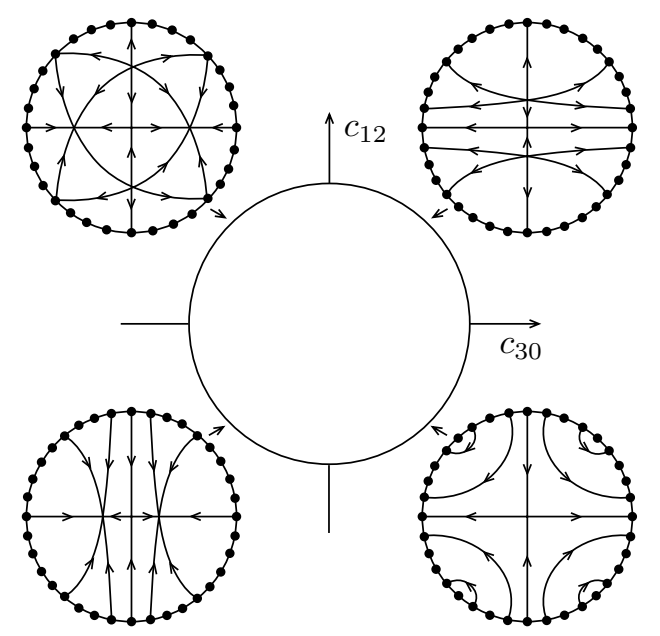

Figure 8 
For case IV we suppose $c_{30}^{2}+d_{03}^{2}=1$. The bifurcation diagram appears in Figure 9.

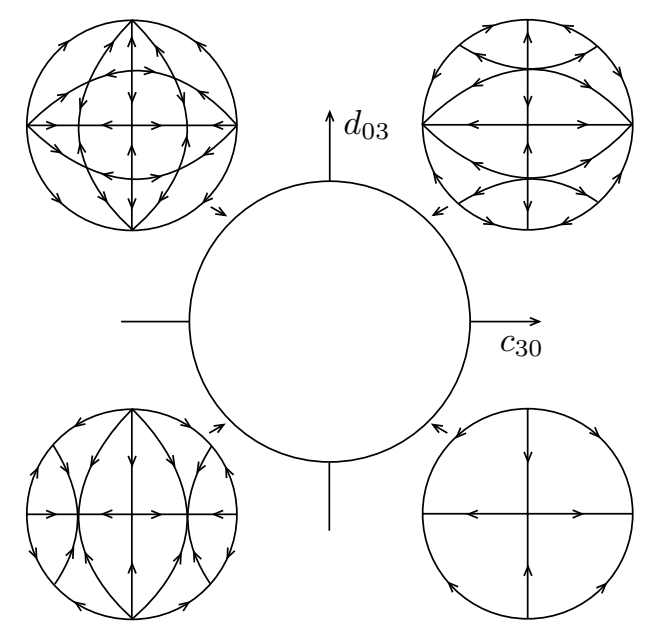

Figure 9

Case $\mathrm{V}$ is zero-dimensional. We have the conditions $c_{30} c_{12}<0$, $d_{30} c_{03}<0, c_{30} d_{21}>0, c_{12} d_{03}>0$ which yields, after scaling, the two cases

$$
\begin{aligned}
& \dot{x}=x+7 x^{3}-3 x y^{2}-4 y^{3} \\
& \dot{y}=-y+4 x^{3}+3 x^{2} y-7 y^{3}
\end{aligned}
$$

and

$$
\begin{aligned}
& \dot{x}=x-7 x^{3}+3 x y^{2}+4 y^{3} \\
& \dot{y}=-y-4 x^{3}-3 x^{2} y+7 y^{3} .
\end{aligned}
$$

Their respective phase portraits appear in Figures 10 and 11.

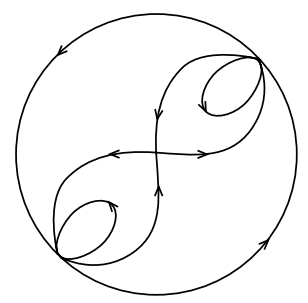

Figure 10 


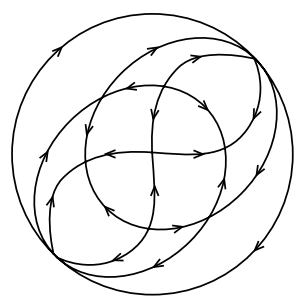

FiguRe 11

For case VI we suppose $c_{03}^{2}+d_{21}^{2}=1$ and $c_{03} \geq 0$. The bifurcation diagram appears in Figure 12. (Case VII is dual.)

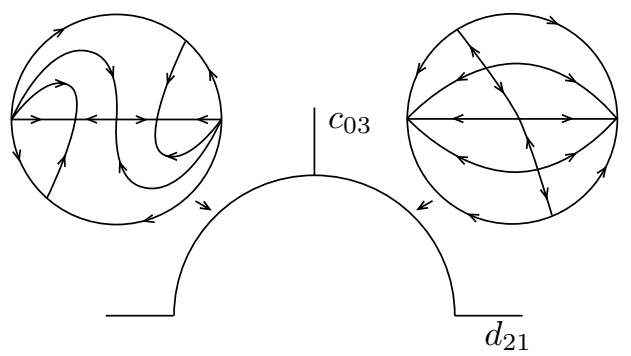

FiguRe 12

Theorem 5.10 ([LL]). The strata of integrable saddles are given by

(A) $\quad d_{21}+3 c_{30}=c_{21}+d_{12}=c_{12}+3 d_{03}=0$,

(B) $\quad c_{21}=d_{12}=c_{30}-3 d_{21}=3 c_{12}-d_{03}$

$$
=3 c_{03} d_{30}-4 d_{21} d_{03}=0 \text {, }
$$

(C) $\quad c_{21}+d_{12}=c_{30} c_{12}-d_{21} d_{03}=c_{30}^{2} c_{03}+d_{30} d_{03}^{2}$

$$
=c_{12}^{2} d_{30}+c_{03} d_{21}^{2}=0 \text {. }
$$

The stratum $(A)$ consists of Hamiltonian systems.

The stratum $(B)$ consists of systems having a Malkin first integral constructed generically from an invariant quartic and an invariant sextic which give a rational first integral.

The stratum $(C)$ consists of reversible systems (called symmetric in $[\mathbf{L L}])$, possibly in the generalized sense of Theorem 5.6 or in the sense below. 
For the substratum $\left(C_{2}\right)$ given by

$$
c_{12}-d_{21}=c_{30}-d_{03}=c_{03}+d_{30}=c_{21}+d_{12}=0
$$

there is no symmtry axis and it cannot be studied by means of Theorem 5.6. The geometry can be explained as follows. We consider the double folding transformation

$$
(u, v) \mapsto\left(x^{2}-y^{2}, x y\right)
$$

The system can then be shown to arise from a new system

$$
\begin{aligned}
& \dot{u}=\left(u^{2}+v^{2}\right)(1+h(u, v)) \\
& \dot{v}=\left(u^{2}+v^{2}\right)(k(u, v)) .
\end{aligned}
$$

When we divide through by the common factor $\left(u^{2}+v^{2}\right)$, the origin is not a critical point. Trajectories passing close to the origin approximate to the lines $v=$ constant and are transformed to trajectories approximating the curves $x y=$ constant. Furthermore the first integral $\phi(u, v)=v+$ $o(|(u, v)|)$ which exists in a neighbourhood of the origin is transformed to a first integral of the original system $\phi\left(x^{2}-y^{2}, x y\right)=x y+o\left(|(x, y)|^{2}\right)$ and so the origin is integrable. Visually, this transformation is equivalent to the 2-1 map $z \mapsto z^{2}$ of the complex plane to itself.

Proposition 5.11. The linearizable saddles described in Theorem 5.9 lie in the stratum $(C)$ for the cases $I-V$ and the Malkin stratum $(B)$ for the cases VI-VII. In the latter case the invariant quartic is reducible, yielding a line through the origin and a cubic.

Finally, we raise some questions which are natural from the phase portraits given in this paper and from the fact that all the examples of linearizable saddles studied in this paper have at least one separatrix which is an invariant algebraic curve.

Conjecture 5.12. A homoclinic loop through an integrable saddle of a real vector field in the plane is an obstruction to linearizability. More generally, any integrable saddle cannot lie on a monodromic graphic.

Question 5.13. Does there exist a complex polynomial system with a nondegenerate linearizable centre at the origin and such that no separatrix of the origin is an algebraic curve? 
This last question is linked with $[\mathbf{Z}]$, where Żołądek considers centres with eigenvalues in $p:-q$ resonances with $p \neq q$. In view of his examples he asks the following question: Does there exist a complex polynomial system with a centre at the origin with eigenvalues in $p:-q$ resonances with $p \neq q$ and such that no separatrix of the origin is an algebraic curve? From Remark 2.9 and the calculations in the previous sections we know that we need not necessarily have both separatrices algebraic. Again this is in contrast to the case of real isochronous centres where the separatrices must be conjugate.

Acknowledgements. The authors are grateful to Dana Schlomiuk for helpful discussions. They thank the referee for pointing to them the theorem of Cartan-CarathThetaodory. The first author thanks the Centre de Recherches Mathématiques for its hospitality in 1995.

\section{References}

[CJ] C. Chicone And M. JACOBs, Bifurcation of critical periods for plane vector fields, Trans. Amer. Math. Soc. 312(2) (1989), 433-486.

[C] C. J. Christopher, Invariant algebraic curves and conditions for a centre, Proc. Roy. Soc. Edinburgh Sect. A 124(6) (1994), 1209-1229.

[CD] C. J. Christopher AND J. Devlin, Isochronous centers in planar polynomial systems, SIAM J. Math. Anal. 28(1) (1997), 162-177.

[CMR] C. J. Christopher, P. Mardešić and C. Rousseau, Normalizable, integrable and linearizable saddle points in complex quadratic systems in $\mathbb{C}^{2}$, in preparation.

[D] G. DARboux, Mémoire sur les équations différentielles algébriques du premier ordre et du premier degré (Mélanges), Bull. Sci. Math. ? (1878), 60-96, 123-144, 151-200.

[De] J. Devlin, Coexisting isochronous and nonisochronous centres, Bull. London Math. Soc. 28(5) (1996), 495-500.

[Du] H. Dulac, Mélanges, Bull. Sci. Math. 32 (1908), 230-252.

[DGS] Y. Dupuis, M. Gagné And D. Schlomiuk, Systèmes quadratiques intégrables avec point de selle, Mémoire de maîtrise de Y. Dupuis, Université de Montréal (1999).

[F] L. FARELL, Systèmes différentiels complexes à centres, Preprint Université de Montréal (1999). 
[LL] Y. R. LiU AND J. B. Li, Theory of values of singular point in complex autonomous differential systems, Sci. China Ser. A 33(1) (1990), 10-23.

[L] W. S. Loud, Behavior of the period of solutions of certain plane autonomous systems near centers, Contributions to Differential Equations 3 (1964), 21-36.

[MMR] P. Mardešić, L. Moser-Jauslin and C. Rousseau, Darboux linearization and isochronous centers with a rational first integral, J. Differential Equations 134(2) (1997), 216-268.

[MRT] P. Mardesic, C. Rousseau and B. Toni, Linearization of isochronous centers, J. Differential Equations 121(1) (1995), 67-108.

[M] J. Moser, "Stable and random motions in dynamical systems", Annals of Mathematics Studies 77, Princeton University Press, Princeton, N. J., 1973.

[P] I. I. PleshKAN, A new method of investigating the isochronicity of a system of two differential equations, Differential Equations 5 (1969), 796-802.

[RT1] C. Rousseau And B. Toni, Local bifurcation of critical periods in vector fields with homogeneous nonlinearities of the third degree, Canad. Math. Bull. 36(4) (1993), 473-484.

[Sa1] M. Sabatini, Quadratic isochronous centers commute, Appl. Math. (Warsaw) 26(3) (1999), 357-362.

[Sa2] M. SABATini, Characterizing isochronous centres by Lie brackets, Differential Equations Dynam. Systems 5(1) (1997), 91-99.

[S1] D. Schlomiuk, Algebraic particular integrals, integrability and the problem of the center, Trans. Amer. Math. Soc. 338(2) (1993), 799-841.

[S2] D. Schlomiuk, Elementary first integrals and algebraic invariant curves of differential equations, Exposition. Math. 11(5) (1993), 433-454.

[SM] C. L. Siegel AND J. K. Moser, "Lectures on celestial mechanics", Die Grundlehren der mathematischen Wissenschaften 187, Springer-Verlag, New York, 1971.

[TY] M. A. TEIXEIRA AND J. YANG, The center-focus problem and reversibility, Preprint, University of Campinas, Brazil (1998).

[Z] H. ŻOŁA̧DEK, The problem of center for resonant singular points of polynomial vector fields, J. Differential Equations 137(1) (1997), 94-118. 
C. Christopher:

School of Mathematics and Statistics

University of Plymouth

Plymouth, Devon PL4 8AA

United Kingdom

E-mail address: C.Christopher@plymouth.ac.uk

C. Rousseau:

Département de Mathématiques et de Statistique, and CRM

Université de Montréal

C. P. 6128 , Succursale Centre-Ville

Montréal, Québec, H3C 3J7

Canada

E-mail address: rousseac@dms.umontreal.ca

Primera versió rebuda el 3 de desembre de 1999, darrera versió rebuda el 22 de setembre de 2000. 